\title{
Evaluation of a DGP Point-of-care Test for Celiac Disease in a Pediatric Population
}

\author{
Claude-Olivier Marti ${ }^{1,2, \neq), *}$, Benoit Fellay ${ }^{1, \ddagger)}$, Annemarie Bürgin-Wolff ${ }^{3}$, Jean-Luc Magnin ${ }^{1}$ Peter Baehler $^{1}$ \\ ${ }^{1}$ Hôpital Cantonal Fribourg, Fribourg, Switzerland \\ ${ }^{2}$ Laboratoire Promed, Marly Switzerland \\ ${ }^{3}$ Coeliakie Institut, Liestal, Switzerland \\ ${ }^{\ddagger}$ Have contributed equally to this work \\ *Corresponding author: claude-olivier.marti@promed-lab.ch
}

Received January 12, 2015; revised January 22, 2015; accepted January 25, 2015

\begin{abstract}
Celiac disease (CD) is a largely undiagnosed immune-mediated enteropathy. A point-of-care test (POCT) could represent a rapid and cost-effective tool on ruling out $\mathrm{CD}$. The objective of this study is to evaluate retrospectively the performance of a POCT based on detection of IgA and IgG antibodies against deamidated gliadins peptides (DGP). The study was performed on 53 children presenting clinical suspicions of CD, all being investigated by intestinal biopsy and standard serology. The performance of POCT was compared to the diagnostic result obtained from the gold standard of histology and serology. 10 children were diagnosed as CD positive by the POCT. Among the 43 children identified as CD negatives, 37 were correctly identified by POCT and 6 were considered as false positives. No false negative results were observed. The POCT yields a sensitivity and a negative predictive value (NPV) of $100 \%$, and a negative likelihood ratio (LR-) of 0 in this selected pediatric population. The high NPV and low LR- ratio indicate that this POCT could be an useful and discriminative tool for excluding CD. This study and the results published so far are promising but need to be confirmed in larger cohort.
\end{abstract}

Keywords: rule-out $C D$, rapid test, children

Cite This Article: Claude-Olivier Marti, Benoit Fellay, Annemarie Bürgin-Wolff, Jean-Luc Magnin, and Peter Baehler, "Evaluation of a DGP Point-of-care Test for Celiac Disease in a Pediatric Population." International Journal of Celiac Disease, vol. 3, no. 1 (2015): 7-11. doi: 10.12691/ijcd-3-1-8.

\section{Introduction}

Celiac disease (CD) is defined as a systemic immunemediated enteropathy induced by dietary gluten (from wheat, barley, rye) in genetically predisposed individuals carrying the HLA-DQ2 and/or DQ8 haplotypes $[1,2,3]$. $\mathrm{CD}$ is characterized by a triggered autoimmune response and subsequent modification of the intestinal integrity, engendering a broad range of clinical presentations $[4,5]$. Its prevalence is approximately $1 \%$ among the European population but shows wide regional differences for unclear reasons (e.g. $0.3 \%$ in Germany, $0.8 \%$ in Switzerland and $2.4 \%$ in Finland) $[6,7,8]$. Different environmental factors such as breast-feeding, cereal consumption or infection have been proposed to influence the risk of developing the disease $[5,9,10]$.

The diagnosis of CD has relied for decades on duodenal biopsy with characteristic histologic pathology as a gold standard. Nowadays, serological markers are a useful diagnostic tool [11]. They have improved substantially in the last two decades and led to the identification of more sensitive and specific antibodies. In parallel, the role of the duodenal biopsy as the "gold standard" has been questioned by a high variability in the histological interpretation. These changes have led to the publication of new guidelines by the European Society for Pediatric Gastroenterology, Hepatology, and Nutrition (ESPGHAN, [2]). The aim of the new guideline was to achieve high diagnostic accuracy and to reduce burden for patients by relying more on genetic and serological tests and therefore decreasing the percentage of patients where biopsy is still required.

IgA anti-tissue transglutaminase (tTG) and the related endomysium antibodies (EMA) are today considered as the most reliable serological markers. The anti-gliadin antibodies (AGA) have been recently replaced by a new generation of tests based on the detection of antibodies against deaminated gliadin peptides (DGP). Recently, serological tests based on detection of antibodies against DGP showed a very good sensitivity and specificity in pediatric populations $[12,13,14]$ Moreover, several studies have shown that IgG anti-DGP have a high specificity and a better sensitivity than IgG anti-tTG in adults and children $[2,15,16]$.

Given that CD is a lifelong condition that can be treated by strict gluten-free diet (GFD), early and rapid diagnosis is mandatory as it allows for faster counselling and treatment, avoiding progression of the condition, decreasing development of comorbidities, and leading to better patient comfort and a reduction in the use of healthcare services in the long term [17]. 
The use of a point-of-care test (POCT) allowing to rapidly rule out $\mathrm{CD}$ in first line primary care setting could be one of the solutions to palliate these needs. Recently, a new POCT based on simultaneous detection of IgA and IgG antibodies against DGP as well as of total IgA, has been shown to have the potential to improve diagnostic screening tool for CD in a pediatric population $[18,19]$.

The aim of this study was to evaluate retrospectively the performance of this novel point-of-care screening test in a pediatric population of 53 children tested for both serological markers (EMA, IgA tTG, IgA/IgG DGP) and intestinal biopsy.

\section{Material and Methods}

\subsection{Patients}

This retrospective study enrolled a cohort of 53 consecutive children (21 females, 32 males; mean age: 5.9, median: 4, range 1-14 years old). Every patient was investigated with serological tests, biopsy and remaining sera were frozen and stored at $-80^{\circ} \mathrm{C}$. All the referred children presented gastrointestinal symptoms (compatible with a suspicion of $\mathrm{CD}$ ) and were examined at the outpatient clinic for pediatric gastroenterology in a secondary referral center (Hôpital cantonal Fribourg) from October 2000 to September 2011. This study was approved by the Ethical committee (Canton de Fribourg, 001/13-CER-FR).

\subsection{Biopsies}

Duodenal biopsies were performed by the pediatric gastroenterologist by upper gastrointestinal endoscopy according to the standard technique [2]. The biopsies were sampled from the bulb up to the distal duodenum. The mucosal biopsy sections were analyzed by an experienced histopathologist to assess for pathologic features of CD that included villous atrophy, crypt hyperplasia and increased intraepithelial lymphocytes (cut-off: > 30 per 100 enterocytes). A diagnosis of CD was graded according to the Oberhuber-Marsh classification system [20].

\subsection{Serum Samples}

Venous blood samplings were drawn by phlebotomy. After centrifugation at $2000 \mathrm{~g}$ for 10 minutes at $20^{\circ} \mathrm{C}$, the sera were aliquoted and sent to the Labor für ZöliakieDiagnostik in Liestal (Switzerland) for serologic analysis and then stored at $-80^{\circ} \mathrm{C}$ until analysis.

\subsection{Routine Assays}

The following assays were performed according to the manufacturer's instructions: 1) IgA anti-endomysium antibodies (EMA) by indirect immunofluorescence technique (IFI, INOVA) 2) IgA anti-tTG enzyme-linked immunosorbent assay (Elia Celikey IgA, Elia Gliadin IgA, Elia Gliadin IgG, Elia Gliadin DP IgA and Elia Gliadin DP IgG; Phadia [now Thermo Fisher Scientific], Freiburg, Germany), 3) prior to March 2010, IgG and IgA Gliadin (AGA) (Elia Celikey, Phadia) were used, and then replaced by IgG and IgA DGP ELISA (Deaminated Gliadin Peptides, Elia Celikey, Phadia), and 4) total IgA by nephelometry (BNII system; Siemens).
The laboratory reference assay cut-off values are: IgA tTG, IgG or IgA AGA or DGP: 7-10 U/ml: greyzone, > 10 $\mathrm{U} / \mathrm{ml}$ positive. IgA anti-endomysium antibiodies: dilution between $1 / 5$ and 1/10: weak positive, $>1 / 20$ positive.

\subsection{Evaluation of the POCT}

All anonymized sera (20 $\mu \mathrm{l})$ were thawed and immediately analyzed using POCT according to the manufacturer's instructions (Simtomax, Augurix, Switzerland). The principle is based on lateral flow immunochromatographic technology using colloidal gold antihuman antibodies. It detects simultaneously the combination of both IgA and IgG antibodies against DGP (line A), as well as total IgA (line B) and an internal positive control (line CT), Figure 1. As mentioned in the Instructions for use (IFU), this test is designed to have the same accuracy using capillary whole blood, venous whole blood, plasma (EDTA or heparinated) or serum.
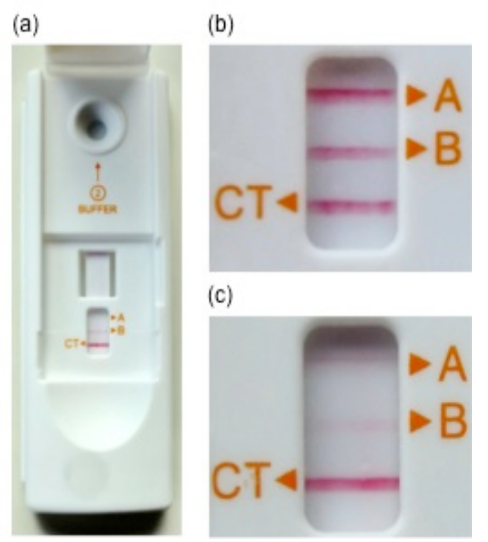

Figure 1. Presentation of the POCT device. a) Result interpretation of the POCT device with the 3 bands: A line corresponds to IgA/IgG DGP, B line to Total IgA, and CT line to control. b) Picture of the test after 10 minutes incubation. Result represents a celiac positive (A line) with normal IgA levels (line B). c) Representation of a false positive result with a thin line A after 10 minutes. This band becomes stronger after 30 minutes of incubation (picture not showed)

The results were visually interpreted after 10 minutes by three qualified laboratory members unaware of the clinical diagnosis, and pictures of each individual test were taken. The results were interpreted as negative (no band), weak positive or positive. All the weak positive tests were repeated using the same protocol.

\subsection{Statistical Analysis}

The results obtained on POCT were compared with the outcome of the laboratory tests and biopsy. The POCT sensitivity, specificity, positive predictive value (PPV), negative predictive value (NPV), and positive and negative likelihood ratios (LR+, LR-) and their corresponding 95\% confidence intervals (CIs) were calculated using the STATA software (version 11; College Station, TX, USA).

\section{Results}

Based on biopsy results, of the 53 selected children, 10 children were diagnosed as CD positive, and 43 children were diagnosed as CD negatives. The distribution of the different groups of the cohort is shown in Table 1. 
Table 1. Summary of the different results among the 53 children

\begin{tabular}{|c|c|c|c|c|c|}
\hline & EMA & $\begin{array}{l}\text { IgA } \\
\text { tTG }\end{array}$ & $\begin{array}{l}\text { IgA/IgG } \\
\text { Gliadin }\end{array}$ & Biopsy & POCT \\
\hline \multicolumn{6}{|l|}{ Celiac } \\
\hline $\mathrm{n}=8^{2)}$ & + & + & + & + & + \\
\hline $\mathrm{n}=2^{3)}$ & - & - & - & + & - \\
\hline
\end{tabular}

Non

Celiac

$\mathrm{n}=37$

$\mathrm{n}=6$

${ }^{1)}$ native or deamidated gliadin; ${ }^{2)}$ on normal diet; ${ }^{3)}$ on GFD; ${ }^{4)}$ weak positive on POCT.

Among the 10 celiac children, 8 patients, with previously undiagnosed coeliac disease, were correctly identified by the POCT. The 8 children were positive for all the serological tests (EMA, IgA tTG, IgA/G DGP) and had biopsies compatible with CD (Marsh 3a-3c, Table 2). All were correctly diagnosed by the POCT.

The band A became weak pink after a 10 minutes incubation time (manufacturer's instruction), questioning the presence or not of a CD positive test. It is interesting to note that the intensity of the line became stronger after 30 minutes.

The total IgA status was correctly identified by POCT for all the patients, for whom the information were available $(\mathrm{n}=44)$. Among the 54 children, no IgA deficiency was found.

Therefore, according to our results, the POCT yields a sensitivity of $100 \%$ (95\%CI: $63.1-100 \%)$ and a specificity of $86 \%$ (95\%CI: $72.1-94.7 \%$ ). The positive and negative predictive values (PPV and NPV) were 57\% (95\%CI: 28.9-82.3\%) and 100\% (95\%CI: 90.5-100\%) respectively.

Table 2. Summary of the results observed between the standard serological tests and POCT

\begin{tabular}{|c|c|c|c|c|c|c|c|c|}
\hline \multirow[b]{2}{*}{ Gender } & \multicolumn{6}{|c|}{ Standard Serology \& Biopsy } & \multicolumn{2}{|c|}{ POCT } \\
\hline & $\begin{array}{c}\text { EMA } \\
\text { titer }\end{array}$ & $\begin{array}{c}\text { Total } \\
\text { IgA } \\
\text { (Reference values) } \\
\end{array}$ & $\begin{array}{l}\text { IgA } \\
\text { tTG }\end{array}$ & $\begin{array}{c}\text { IgA } \\
\text { AGA/DGP }\end{array}$ & $\begin{array}{c}\text { IgG } \\
\text { AGA/DGP }\end{array}$ & $\begin{array}{c}\text { Biopsy } \\
\text { (Marsh) }\end{array}$ & $\begin{array}{c}\text { IgA/IgG } \\
\text { DGP }\end{array}$ & $\begin{array}{c}\text { Total } \\
\text { IgA }\end{array}$ \\
\hline \multicolumn{7}{|c|}{ Celiac } & & \\
\hline M & $1 / 320$ & $2(0.35-2)$ & $>100$ & $\begin{array}{c}78 \\
\text { (DGP) }\end{array}$ & $\begin{array}{c}60 \\
\text { (DGP) }\end{array}$ & За & + & + \\
\hline M & $1 / 80$ & $\mathrm{nd}^{1)}$ & 130 & $\begin{array}{c}8 \\
\text { (DGP) }\end{array}$ & $\begin{array}{c}11 \\
\text { (DGP) }\end{array}$ & 3c & + & $+/-$ \\
\hline $\mathrm{F}$ & $1 / 320$ & $0.8(0.35-2)$ & $>100$ & $\begin{array}{c}133 \\
\text { (DGP) }\end{array}$ & $\begin{array}{l}>100 \\
(\mathrm{DGP})\end{array}$ & $3 b$ & + & $+/-$ \\
\hline $\mathrm{F}$ & $1 / 160$ & $1.24(0.35-2)$ & $>100$ & $\begin{array}{c}12 \\
(\mathrm{AGA})\end{array}$ & $\begin{array}{c}34 \\
(\mathrm{AGA})\end{array}$ & За & + & $+/-$ \\
\hline M & $1 / 320$ & nd & 150 & $\begin{array}{c}11 \\
\text { (AGA) }\end{array}$ & $\begin{array}{c}27 \\
(\mathrm{AGA})\end{array}$ & $3 c$ & + & $+/-$ \\
\hline $\mathrm{F}$ & $1 / 160$ & $1.2(0.4-2)$ & $>100$ & $\begin{array}{c}14 \\
\text { (AGA) }\end{array}$ & $\begin{array}{c}14 \\
\text { (AGA) }\end{array}$ & 3c & + & + \\
\hline $\mathrm{F}$ & $1 / 640$ & $2.24(0.7-4)$ & 105 & $\begin{array}{c}15.6 \\
\text { (AGA) }\end{array}$ & $\begin{array}{c}105 \\
\text { (AGA) }\end{array}$ & $3 b$ & + & $+/-$ \\
\hline M & $1 / 1280$ & $1.83(0.7-4)$ & nd & $\begin{array}{c}51 \\
\text { (AGA) }\end{array}$ & $\begin{array}{c}85 \\
\text { (AGA) }\end{array}$ & 3a & + & $+/-$ \\
\hline \multicolumn{7}{|c|}{ Celiac under Gluten Free Diet } & & \\
\hline M & - & nd & 0.5 & $\begin{array}{c}0.4 \\
\text { (DGP) }\end{array}$ & $\begin{array}{c}1.2 \\
\text { (DGP) }\end{array}$ & $3 c$ & - & $+/-$ \\
\hline M & - & nd & 4.5 & $\begin{array}{c}0.6 \\
\text { (DGP) }\end{array}$ & $\begin{array}{c}0.8 \\
\text { (DGP) }\end{array}$ & $3 b-3 c$ & - & $+/-$ \\
\hline \multicolumn{7}{|c|}{ POCT false positive results } & & \\
\hline M & - & $1.12(0.3-1.6)$ & 0.4 & $\begin{array}{c}0.8 \\
(\mathrm{DGP})\end{array}$ & $\begin{array}{c}2.3 \\
(\mathrm{DGP})\end{array}$ & 0 & $+/-$ & $+/-$ \\
\hline $\mathrm{F}$ & - & $0.64(0.4-2)$ & 0.2 & $\begin{array}{c}0.8 \\
\text { (DGP) }\end{array}$ & $\begin{array}{c}0.7 \\
\text { (DGP) }\end{array}$ & 0 & $+/-$ & $+/-$ \\
\hline M & - & $1.05(0.7-4)$ & 0.2 & $\begin{array}{c}1.4 \\
\text { (DGP) }\end{array}$ & $\begin{array}{c}1.1 \\
\text { (DGP) }\end{array}$ & 0 & $+/-$ & + \\
\hline M & - & $1.13(0.4-2.5)$ & 0.1 & $\begin{array}{c}0.6 \\
\text { (DGP) }\end{array}$ & $\begin{array}{c}3.4 \\
\text { (DGP) }\end{array}$ & 0 & $+/-$ & $+/-$ \\
\hline $\mathrm{F}$ & - & $0.97(0.4-2.5)$ & 0.2 & $\begin{array}{c}0.9 \\
\text { (DGP) }\end{array}$ & $\begin{array}{c}5.3 \\
\text { (DGP) }\end{array}$ & 0 & $+/-$ & $+/-$ \\
\hline M & - & $0.3(0.35-2)$ & 0 & $\begin{array}{c}0.3 \\
\text { (DGP) }\end{array}$ & $\begin{array}{c}4.2 \\
\text { (DGP) }\end{array}$ & 0 & $+/-$ & $+/-$ \\
\hline
\end{tabular}

nd: not determined.

Of the 10 celiac children, 2 children were previously diagnosed for $\mathrm{CD}$, and were having their first yearly follow-up for compliance to GFD by the time of the laboratory serology analyses. All the serological tests were negative one year after the instauration of the GFD as well as the POCT results.

Among the 43 non-celiac children, 37 were correctly diagnosed by POCT. However, the POCT yields 6 false positive results (14\%). A weak positive band corresponding to IgA and IgG DGP antibodies (band A) was observed for these children and confirmed by retesting the sera once again with a new test (Table 2).

Likelihood ratios are an alternative way of describing the performance of a diagnostic test and could be uses to calculate the probability of disease after a positive or negative test. However, in contrast to PPV and NPV, it does not depend on the prevalence of the disease. Here, the negative LR (LR-) is of 0 (95\%CI: 0.004-0.97) and the positive LR (LR+) is of 7.14 (95\%CI: 3.09-13.23) (Table 3). 
Table 3. POCT results compared to diagnosis of celiac disease based on standard serology and biopsy

\begin{tabular}{|c|c|c|c|c|}
\cline { 3 - 5 } \multicolumn{2}{c|}{} & \multicolumn{2}{c}{ Serological tests \& Biopsy } & \multicolumn{1}{c}{ Total } \\
\hline \multirow{2}{*}{ POCT } & + & $8^{*}$ & 6 & 14 \\
\cline { 2 - 5 } & - & 0 & 37 & 37 \\
\hline
\end{tabular}

* the two children under the GFD were excluded.

\section{Discussion}

Although the diagnosis of CD largely improved in the past two decades, the prevalence of the disease is still raising and many patients remain undiagnosed [21,22]. This is due in part, to difficulties of interpreting mild or atypical symptoms of CD by the primary care physicians $[23,24]$. A large retrospective study showed that a rapid diagnosis is associated with a significant overall medical cost reduction attributable to decrease number of doctoral office visits, laboratory services, imaging support services, and endoscopy procedures [25,26,27,28].

Primary care physicians play an important role in the screening of patients presenting mostly nonspecific symptoms related to CD. An accurate POCT could facilitate the differential diagnostic procedure and hence reorienting POCT negative patient towards other pathologies, or it can improve the investigation of POCT positive patients according to the new ESPGAN guidelines $[2,29]$. This first line screening tool has to be used by health professionals who could interpret and handle properly unexpected or equivocal results.

Recently, Mooney et al pointed out the lack of comparison between the above-mentioned POCT and the gold standard of histology [30]. This study provides the first evidences that this POCT is useful when compared to the gold standard of histology. It is noteworthy that all children of this study were both biopsied and tested by standard serological analyses.

The results obtained with this POCT showed an excellent sensitivity and specificity. Nevertheless, we are aware that the limited number of CD patients has an impact on the results and may explain the large 95\% Confidence Interval (CI) for sensitivity (66-100\%); in fact, one discordant result changes considerably the sensitivity. However, two recent studies have shown comparable sensitivity and specificity in larger groups, including pediatric and adult patients. Bienvenu et al. demonstrated an acceptable discriminative value in a large pediatric population (250 children) composed of patients with CDrelated symptoms or at high risk for CD. The test yielded a sensitivity of 93.1\%, and a NPV of 99.1\% [19]. Benkebil et al. reported a slightly lower sensitivity (78.9\%) but comparable specificity (95.7\%) with an adult population composed of patients visiting a gastroenterology outpatient clinic [18].

Overall, among the 53 children tested, the POCT yielded 47 accurate results, no false negative results, and 6 false positive results. Although a high sensitivity is preferred for screening tests, a condition having a low prevalence will yield a high number of false-positive results. Since the prevalence of CD in Switzerland is about $0.75 \%$ [8], a higher fraction of false positive results could be predicted with this test [30]. Thus, all positive results need further investigations by standard serological testing and referring the patient to a pediatric gastroenterologist. Furthermore, a standard serological approach for quantitative testing remains indispensable for further evaluation, follow-up and control of compliance of GFD in CD patients [18].

From a practical point of view we observed that the reading of the bands is sometimes difficult due to their weak intensity. The homogeneity of the bands as well as the reading conditions (lightning) are important for interpretation. Consequently, it remains essential to perform this POCT by trained individuals familiar to the use of POCT devices.

Self-testing using POCT based on the detection of autoantibodies (tTG or DGP) is already available in commercial shops in Europe, and it raises major concerns. Indeed, individuals who self-diagnose CD based on this test may start a GFD without consulting their primary care physician or a specialist for a correct diagnostic workup. Moreover, the ESPGAN guidelines highlight the need for test interpretation to be done by trained individuals rather than by lay people $[2,30]$.

\section{Conclusion}

Taken together, our data show a good correlation between standard serological tests, biopsy and the POCT results. The high negative predictive value and low LRratio indicate that this POCT could be a useful and discriminative tool for excluding celiac disease. It appears however that our study and the results published so far need to be confirmed in a larger cohort of pediatric patient, in order to confirm this high NPV resulting from high sensitivity and specificity.

\section{Acknowledgements}

We would like to thank Christine Schaflinger from the Coeliakie Institut, Liestal for her help and work.

\section{Conflict of Interest}

Dr. Marti and Dr. Fellay have received a travel grant (flight tickets only) from Augurix. The remaining authors declare that there are no conflicts of interest.

\section{List of Abbreviations}

AGA: anti-gliadin antibody; CD: celiac disease; CIs: confidence intervals; DGP: deamidated gliadin peptide; EDTA: Ethylenediaminetetraacetic acid; ELISA: enzymelinked immunosorbent assay; EMA: endomysium antibodies; ESPGHAN: European Society of Pediatric Gastroenterology, Hepatology and Nutrition; GFD: gluten free diet; HLA: human leukocyte antigen; IFI: indirect immunofluorescence technique; IFU: instruction for use; IgA: immunoglobulin A; IgG: immunoglobulin G; LR: likelihood ratio; NPV: negative predictive value; POCT: point-of-care test; tTG: tissue transglutaminase; PPV: positive predictive value. 


\section{References}

[1] A. Fasano, I. Berti, T. Gerarduzzi, T. Not, R.B. Colletti, S. Drago, Y. Elitsur, P.H. Green, S. Guandalini, I.D. Hill, M. Pietzak, A. Ventura, M. Thorpe, D. Kryszak, F. Fornaroli, S.S. Wasserman, J.A. Murray, K. Horvath, Prevalence of celiac disease in at-risk and not-at-risk groups in the United States: a large multicenter study. Arch Intern Med 163 (2003) 286-292.

[2] S. Husby, S. Koletzko, I.R. Korponay-Szabo, M.L. Mearin, A. Phillips, R. Shamir, R. Troncone, K. Giersiepen, D. Branski, C. Catassi, M. Lelgeman, M. Maki, C. Ribes-Koninckx, A. Ventura, K.P. Zimmer, European Society for Pediatric Gastroenterology, Hepatology, and Nutrition guidelines for the diagnosis of coeliac disease. J Pediatr Gastroenterol Nutr 54 (2012) 136-160.

[3] A. Fasano, C. Catassi, Clinical practice. Celiac disease. N Engl J Med 367 (2012) 2419-2426.

[4] N. Gujral, H.J. Freeman, A.B. Thomson, Celiac disease: prevalence, diagnosis, pathogenesis and treatment. World $\mathrm{J}$ Gastroenterol 18 (2012) 6036-6059.

[5] B. Meresse, G. Malamut, N. Cerf-Bensussan, Celiac disease: an immunological jigsaw. Immunity 36 (2012) 907-919.

[6] W. Kratzer, M. Kibele, A. Akinli, M. Porzner, B.O. Boehm, W. Koenig, S. Oeztuerk, R.A. Mason, R. Mao, M.H. Haenle, Prevalence of celiac disease in Germany: A prospective follow-up study. World J Gastroenterol 19 (2013) 2612-2620.

[7] A. Parnanen, K. Kaukinen, S. Helakorpi, A. Uutela, M.L. Lahdeaho, H. Huhtala, P. Collin, M. Maki, K. Kurppa, Symptomdetected and screen-detected celiac disease and adult height: a large cohort study. Eur J Gastroenterol Hepatol 24 (2012) 10661070.

[8] R. Rutz, E. Ritzler, W. Fierz, D. Herzog, Prevalence of asymptomatic celiac disease in adolescents of eastern Switzerland. Swiss Med Wkly 132 (2002) 43-47.

[9] T. Pozo-Rubio, A. Capilla, J.R. Mujico, G. de Palma, A. Marcos, Y. Sanz, I. Polanco, M.D. Garcia-Novo, G. Castillejo, C. RibesKoninckx, V. Varea, F. Palau, L. Ortigosa, L. Pena-Quintana, E. Nova, Influence of breastfeeding versus formula feeding on lymphocyte subsets in infants at risk of coeliac disease: the PROFICEL study. Eur J Nutr 52 (2013) 637-646.

[10] T. Pozo-Rubio, M. Olivares, E. Nova, G. De Palma, J.R. Mujico, M.D. Ferrer, A. Marcos, Y. Sanz, Immune development and intestinal microbiota in celiac disease. Clin Dev Immunol 2012 (2012) 654143.

[11] A. Burgin-Wolff, B. Mauro, H. Faruk, Intestinal biopsy is not always required to diagnose celiac disease: a retrospective analysis of combined antibody tests. BMC Gastroenterol 13 (2013) 19.

[12] S. Amarri, P. Alvisi, R. De Giorgio, M.C. Gelli, R. Cicola, F. Tovoli, R. Sassatelli, G. Caio, U. Volta, Antibodies to Deamidated Gliadin Peptides: An Accurate Predictor of Coeliac Disease in Infancy. J Clin Immunol (2013).

[13] M. Ankelo, V. Kleimola, S. Simell, O. Simell, M. Knip, E. Jokisalo, M. Tarkia, A. Westerlund, Q. He, M. Viander, J. Ilonen, A.E. Hinkkanen, Antibody responses to deamidated gliadin peptide show high specificity and parallel antibodies to tissue transglutaminase in developing coeliac disease. Clin Exp Immunol 150 (2007) 285-293.

[14] K. Giersiepen, M. Lelgemann, N. Stuhldreher, L. Ronfani, S. Husby, S. Koletzko, I.R. Korponay-Szabo, Accuracy of diagnostic antibody tests for coeliac disease in children: summary of an evidence report. J Pediatr Gastroenterol Nutr 54 (2012) 229-241.

[15] E. Liu, M. Li, L. Emery, I. Taki, K. Barriga, C. Tiberti, G.S. Eisenbarth, M.J. Rewers, E.J. Hoffenberg, Natural history of antibodies to deamidated gliadin peptides and transglutaminase in early childhood celiac disease. J Pediatr Gastroenterol Nutr 45 (2007) 293-300.

[16] E. Schwertz, F. Kahlenberg, U. Sack, T. Richter, M. Stern, K. Conrad, K.P. Zimmer, T. Mothes, Serologic assay based on gliadin-related nonapeptides as a highly sensitive and specific diagnostic aid in celiac disease. Clin Chem 50 (2004) 2370-2375.

[17] L.A. Mizen, M.L. Macfie, L. Findlay, S.A. Cooper, C.A. Melville, Clinical guidelines contribute to the health inequities experienced by individuals with intellectual disabilities. Implement Sci 7 (2012) 42.

[18] C.C. Bekebil F, Anghel SI, Besson Duvanel C, Schappi MG, Diagnostic accuracy of a new point-of-care screening assay for celiac disease. World J Gastroenterol in press (2013).

[19] F. Bienvenu, C. Besson Duvanel, C. Seignovert, P. Rouzaire, A. Lachaux, J. Bienvenu, Evaluation of a point-of-care test based on deamidated gliadin peptides for celiac disease screening in a large pediatric population. Eur J Gastroenterol Hepatol 24 (2012) 14181423.

[20] G. Oberhuber, G. Granditsch, H. Vogelsang, The histopathology of coeliac disease: time for a standardized report scheme for pathologists. Eur J Gastroenterol Hepatol 11 (1999) 1185-1194.

[21] I. Brusca, A. Carroccio, E. Tonutti, D. Villalta, R. Tozzoli, M. Barrale, F.M. Sarullo, P. Mansueto, S.M. Chiusa, G. Iacono, N. Bizzaro, The old and new tests for celiac disease: which is the best test combination to diagnose celiac disease in pediatric patients? Clin Chem Lab Med 50 (2012) 111-117.

[22] E. Tonutti, D. Visentini, A. Picierno, N. Bizzaro, D. Villalta, R. Tozzoli, G. Kodermaz, A. Carroccio, G. Iacono, S. Teresi, S.M. La Chiusa, I. Brusca, Diagnostic efficacy of the ELISA test for the detection of deamidated anti-gliadin peptide antibodies in the diagnosis and monitoring of celiac disease. J Clin Lab Anal 23 (2009) 165-171.

[23] M. Ravikumara, V.K. Nootigattu, B.K. Sandhu, Ninety percent of celiac disease is being missed. J Pediatr Gastroenterol Nutr 45 (2007) 497-499.

[24] K. Kaukinen, K. Lindfors, P. Collin, O. Koskinen, M. Maki, Coeliac disease--a diagnostic and therapeutic challenge. Clin Chem Lab Med 48 (2010) 1205-1216.

[25] P.H. Green, A.I. Neugut, A.J. Naiyer, Z.C. Edwards, S. Gabinelle, V. Chinburapa, Economic benefits of increased diagnosis of celiac disease in a national managed care population in the United States. J Insur Med 40 (2008) 218-228.

[26] P.H.R. Green, S.N. Stavropoulos, S.G. Panagi, S.L. Goldstein, D.J. McMahon, H. Absan, A.I. Neugut, Characteristics of adult celiac disease in the USA: results of a national survey. Am J Gastroenterol 96 (2001) 126-131.

[27] E. Sugai, H. Vazquez, F. Nachman, M.L. Moreno, R. Mazure, E. Smecuol, S. Niveloni, A. Cabanne, Z. Kogan, J.C. Gomez, E. Maurino, J.C. Bai, Accuracy of testing for antibodies to synthetic gliadin-related peptides in celiac disease. Clin Gastroenterol Hepatol 4 (2006) 1112-1117.

[28] K. Conrad, D. Roggenbuck, A. Ittenson, D. Reinhold, T. Buettner, M.W. Laass, A new dot immunoassay for simultaneous detection of celiac specific antibodies and IgA-deficiency. Clin Chem Lab Med 50 (2012) 337-343.

[29] M. Barak, O. Rozenberg, P. Froom, M. Grinberg, D. Reginashvili, C. Henig, A. Pacht, A. Lerner, Challenging our serological algorithm for celiac disease (CD) diagnosis by the ESPGHAN guidelines. Clin Chem Lab Med 51 (2013) e257-259.

[30] P.D. Mooney, M. Kurien, D.S. Sanders, Simtomax, a novel point of care test for coeliac disease. Expert Opin Med Diagn 7 (2013) 645-651. 